\title{
Analyzing Human Factors Contributing to the Explosion in Texas Tech University Laboratory
}

\author{
Nur Azizah Arryanie Morshidi ${ }^{1}$, Haziqah Pethie @ Petihie ${ }^{1}$, Norafneeza Norazahar*1,2 \\ ${ }^{1}$ Faculty of Chemical and Energy Engineering, Universiti Teknologi Malaysia, 81310 Johor Bahru, Johor, \\ Malaysia \\ ${ }^{2}$ Centre of Hydrogen Energy, Institute of Future Energy, Universiti Teknologi Malaysia, 81310 Johor Bahru, \\ Johor, Malaysia \\ *Corresponding author: norafneeza@utm.my
}

\section{Article History}

Received: March 27, 2018

Received in revised form: May 27, 2018

Accepted: June 06, 2018

Published: July 30, 2018

\begin{abstract}
This paper presents the use of human factor analysis and classification system (HFACS) for the explosion in the Texas Tech University laboratory. Human factor issues in the university laboratory were assessed according to four categories in HFACS: unsafe acts, precondition for unsafe acts, unsafe supervision, and organizational influences. The assessment showed that the student committed many errors due to precondition for unsafe acts, in particular no physical hazard evaluation required prior to conducting the experiment. Inadequate supervision in the university laboratory caused the presence of precondition for unsafe acts among students. The trajectory of human factor issues in the categories of unsafe acts, precondition for unsafe acts, and unsafe supervision pointed to the university's organization. The human factor issues were analyzed according to three subcategories in organizational influences: resource management, organizational climate, and organizational process. The use of HFACS in the explosion in the Texas Tech University laboratory could demonstrate the sequence of failures and human factors leading to the accident.
\end{abstract}

Keywords: HFACS, human factors, organizational factors, university laboratory, unsafe acts.

\subsection{INTRODUCTION}

A graduate student of Texas Tech University (TTU) was involved in the laboratory incident on January $7^{\text {th }}, 2010$. According to the U.S. Chemical Safety Board (CSB) [10], the explosion caused him to lose three fingers on his left hand, burned both his hands and face, and injured his eyes. The student was handling nickel hydrazine perchlorate (NHP), which is an energetic and explosive compound, as part of his research work.

The investigation conducted by the CSB [9] revealed two possible causes: poor communication on the limitation of NHP and no risk assessment on NHP prior to conducting the experiment. The principle investigators (PIs) failed to prove that they informed the student regarding the limitation of NHP prior to performing the experiment. Based on the investigation report, both the student and the PIs did not conduct physical hazard evaluation and risk assessment on NHP [9]. As a consequence, the student decided to increase the amount of NHP without consulting the PIs.

Both human and organizational factors are involved in the laboratory incident at TTU. The investigation report briefly discusses the human and organizational factors leading to the poor safety and the laboratory incident [9], [10]. Failure to identify the organizational factor could miss the chance to improve the laboratory and its safety management system [5].

The objective of this paper is to exercise the use of human factor analysis and classification system (HFACS) in an accident. This paper identifies human factor issues contributing to the laboratory incident at TTU. This paper uses the investigation report prepared by the CSB [9] as a main reference for the assessment of human factors. 
This paper presents the HFACS and its approach in Section 2. Failures and human factor issues in the explosion of TTU laboratory are assessed in Section 3. Finally, Section 4 concludes the assessment of human factors in the university laboratory.

\subsection{METHODOLOGY}

Human factor analysis and classification system (HFACS) was introduced by Shappell and Wiegmann [7] based on the Swiss cheese model [6]. The objective of HFACS is to identify failures and human factors leading to incidents and accidents. HFACS can provide an understanding of the series of events affected by human factors. HFACS has been applied in commercial aviation, rail, and shipping industries, as well as medical sectors $[8,1,4,3,2]$.

HFACS consists of four categories for identifying human factor issues, which are unsafe acts, precondition for unsafe acts, unsafe supervision, and organizational influences. Unsafe act is usually known as active failure. Precondition for unsafe acts, unsafe supervision, and organizational influences are classified as latent failures, which are not noticed until the incident or accident happened. All categories are further discussed in Section 2.1 to 2.4.

\subsection{Unsafe Acts}

Unsafe acts are often associated with the individual involved in the incident or accident. According to Shappell and Wiegmann [7], unsafe acts can be divided into two subcategories, which are errors and violations. Three types of errors introduced in HFACS are skill-based, decision, and perceptual errors. There are two types of violations, which are routine and exceptional violations. Both errors and violations are active failures resulting from the precondition for unsafe acts.

\subsection{Precondition for Unsafe Acts}

Preconditions for unsafe acts consist of three subcategories: substandard conditions of individuals, substandard practices of individuals, and environmental factors. The substandard conditions of individual include both physical and mental states of individuals in the working environment. Examples of human factors leading to poor mental state of individuals are distraction, fatigue, and loss of situational awareness. The substandard practices of individuals have two elements: crew resource management and individual readiness [7].

\subsection{Unsafe Supervision}

The precondition for unsafe acts is often caused by poor supervision. HFACS divides unsafe supervision into four subcategories: inadequate supervision, planned inappropriate operations, failed to correct problems, and supervisory violations. Failure to provide training and track individuals' performance working for the specific tasks can be assigned to the subcategory of inadequate supervision. More examples for each subcategory of unsafe supervision are available in the work by Shappell and Wiegmann [7].

\subsection{Organizational Influences}

An organization is responsible for managing and implementing systems associated with tasks. Thus, the organization could influence the individual's performance in completing the tasks. HFACS classifies organizational influences into three subcategories, which are resource management, organizational climate, and organizational process. Resource management focuses on both financial and human resource management [7]. Working environment and safety culture are examples of organizational climate. Organizational process may include documentation, instructions, safety programs, and risk management.

\subsection{RESULTS AND DISCUSSION}

The human factors and failures leading to the explosion of the Texas Tech University laboratory were assessed from the unsafe acts category to precondition for unsafe acts, unsafe supervision, and organizational influences categories. The human factor issues and failures are presented in Section 3.1 to 3.4. 


\subsection{Unsafe Acts}

Referring to the CSB [9], the student was considered as an actor in the event of explosion in the university laboratory. The student's unsafe acts were assessed based on HFACS. Fig. 1 shows both skill-based and decision errors leading to the explosion.

The skill-based errors are due to lack of training prior to handling energetic or explosive compound. The student reported that he did not receive formal safety training on physical hazard evaluation for NHP. He studied NHP by reviewing many research papers by himself [9]. Lack of safety training left the student without the knowledge of proper handling of NHP, as well as physical hazards and risks of NHP.

According to the investigation conducted by the CSB [9], the student made the decision by himself. The student did not seek advice or information from the PIs to increase the amount of NHP. The limit of NHP use is $100 \mathrm{mg}$. The student decided to prepare many batches of NHP that could fully characterize it.

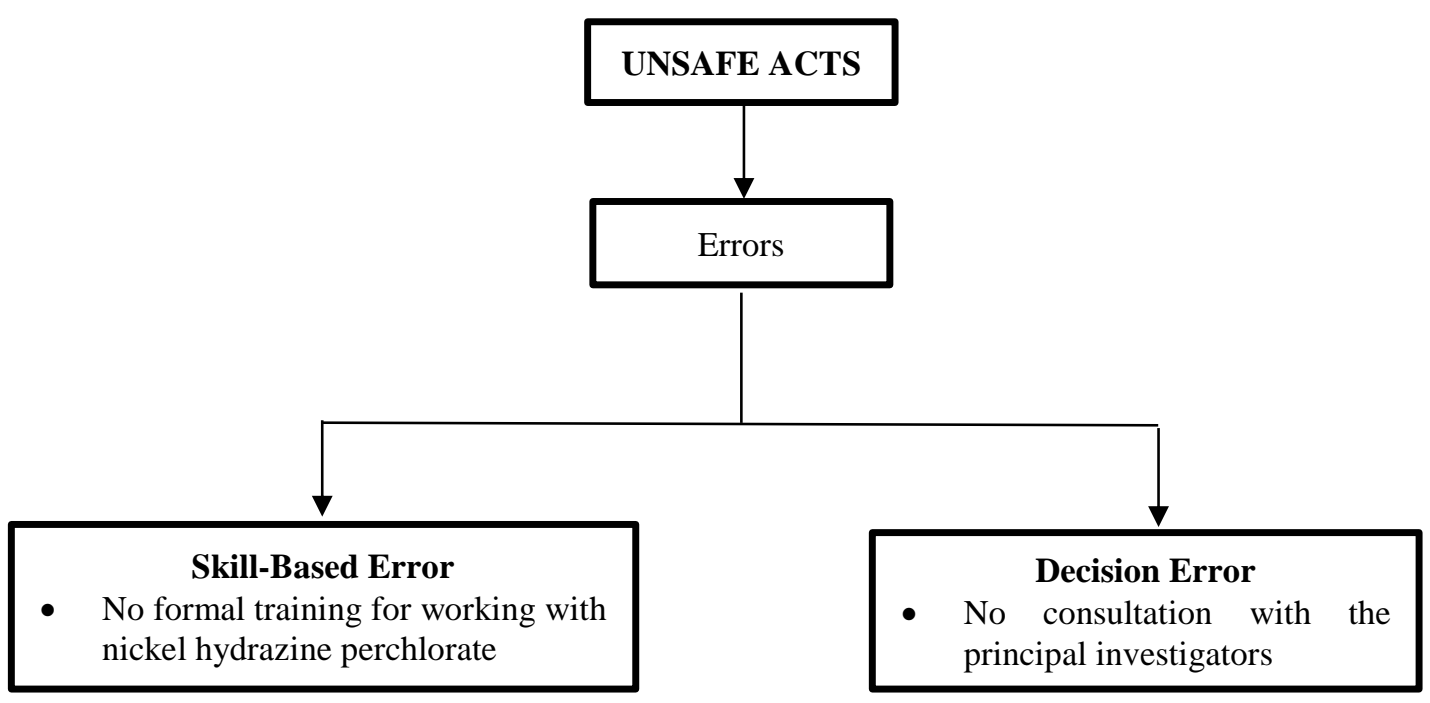

Figure 1. Active failures identified in the university laboratory

\subsection{Precondition for Unsafe Acts}

Fig. 2 presents the precondition for unsafe acts of the student working in the university laboratory. The precondition for unsafe acts was assessed for one subcategory only, which was the substandard practices of students. No information was identified for the subcategory of substandard conditions of students [9].

The first substandard practiced by the student in the university laboratory was the requirement to wear personal protective equipment (PPE). Wearing PPE in the university laboratory is considered as a personal choice as reported by a few individuals to the CSB [9]. Students wore PPE when they perceived the experiment or research work is too risky. The student's eyes were injured in the explosion because he did not wear the safety goggles back after returning to the university laboratory [9].

The limitation of NHP was only communicated verbally. The information regarding limitation was passed from the senior students to the new students working in the university laboratory. There was also no written procedure for the limitation of NHP provided by the department and university. The student did not communicate with the PIs prior to increasing the quantity of NHP because there was no written policy or procedure to do so.

The university laboratory did not have formal hazards and risks evaluation prior to conducting experiments. The university only provided chemical health assessment due to chemical exposure to students working in the laboratory. However, the university failed to equip the laboratory with physical hazard evaluation for energetic or explosive compounds. 


\section{PRECONDITION FOR}

UNSAFE ACTS

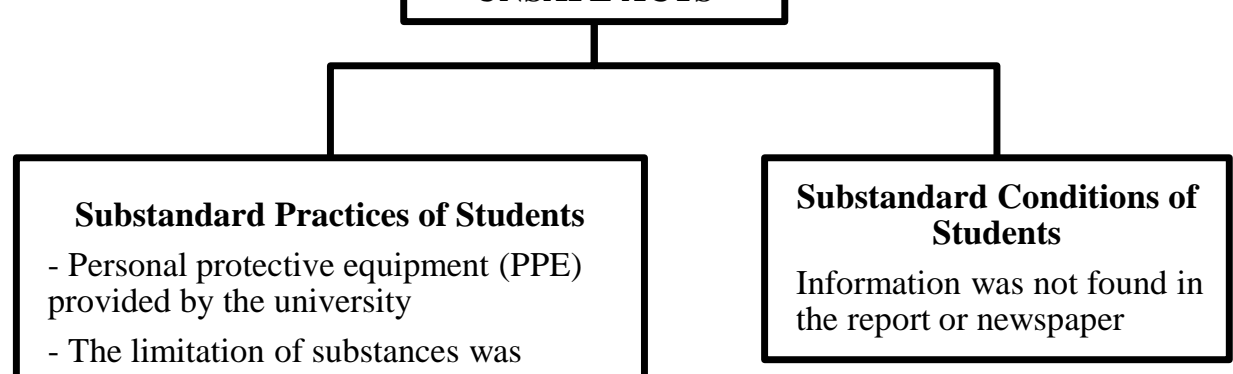
communicated verbally

- No written policy or procedure in the laboratory

- No formal hazard evaluation was conducted by the university

- No formal documentation for safety in the university laboratory

Figure 2. Latent failures due to substandard practices

\subsection{Unsafe Supervision}

The precondition for unsafe acts presents due to unsafe supervision in the university laboratory. Based on the investigation report [9], the substandard practices by students were caused by inadequate supervision in the university laboratory. No training was provided by the university management to students working in the laboratory as stated in Fig. 3. Students reported to the investigation team that they had to find techniques and procedures for handling NHP without a proper guidance [9].

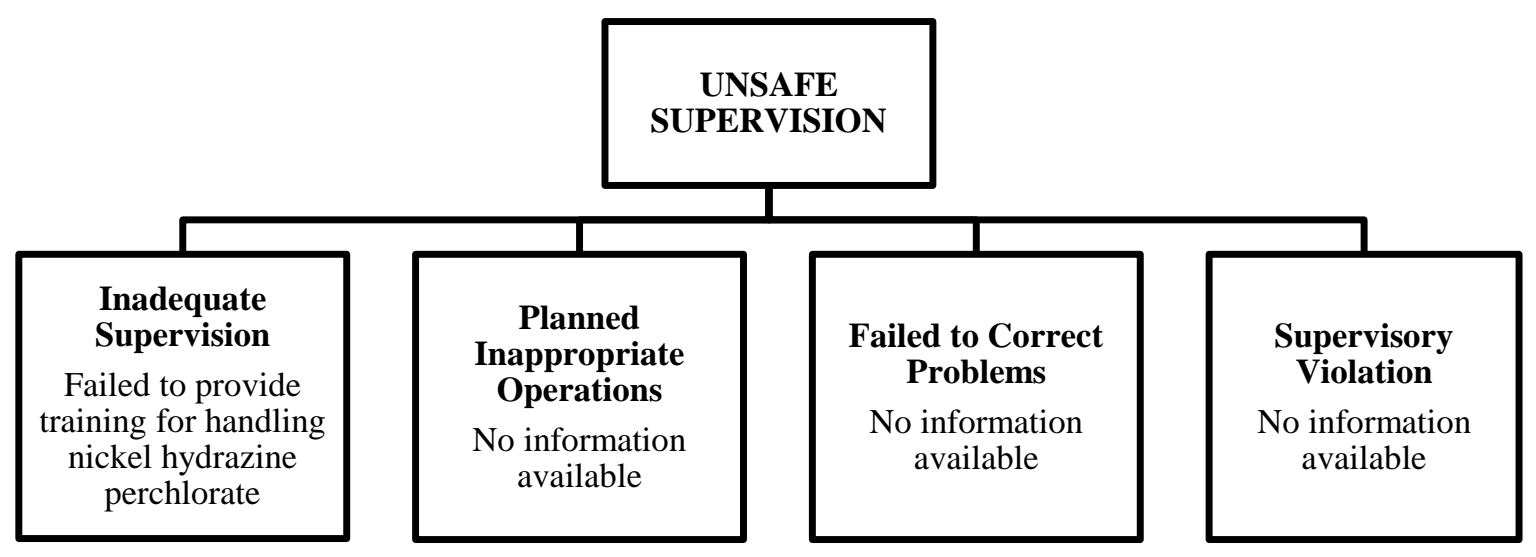

Figure 3. Latent failures due to inadequate supervision in the university laboratory

\subsection{Organizational Influences}

The investigation conducted by the CSB [9] stated that the deficiency of university's organization to manage safety systematically led to the explosion on January $7^{\text {th }}, 2010$. Fig. 4 presents latent failures and human factors in the organizational level. Each subcategory has the human factor issues and failures.

There are many drawbacks in the resource management of the university [9]. The human factor issues in the subcategory of resource management are the failure to hire or assign university staff for tracking and assessing laboratory safety training, updating and enforcing chemical hygiene plan, reviewing safety protocol and procedure in the laboratory, and enforcing policy on handling chemicals. 
Students' safety practices depend on the university's organizational climate. If the university made a serious effort on safety, the students would have conformed to the safety rules and regulations in the laboratory. In the subcategory of organizational climate, the university laboratory was not provided with a tracking system for incidents and near misses occurred in the university laboratory. To make the situation of the university laboratory worse, many incidents were not documented in the safety management system of university and thus, they were not communicated to new students working in the laboratory. The incident in 2007 involving students was not informed to new students [9].

Organizational process involves both the university's organization and students working in the laboratory. The human factor issues related to organizational process are the university's failures to provide safety management systems of the university laboratory, physical hazard evaluation in the OSHA Laboratory Standard, and guidance for determining and mitigating risks of chemicals to students working in the laboratory.

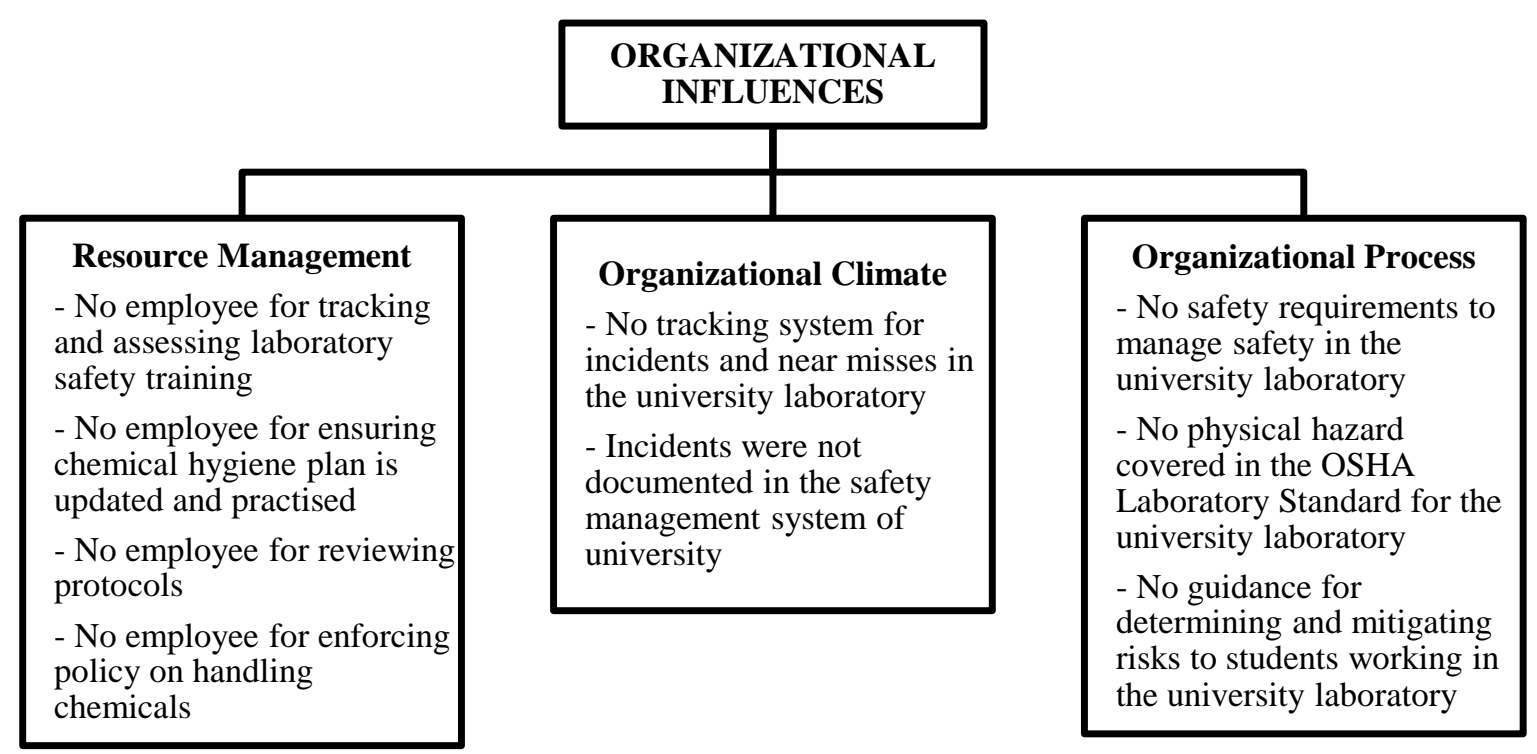

Figure 4. Latent failures due to poor safety management of the university laboratory

\subsection{CONCLUSION}

The explosion in a laboratory of Texas Tech University causing serious injuries to one student reveals the weakness of the university's organization to manage safety systematically. There are many human factor issues in the university's organization based on the investigation report of the CSB [9] supported by the assessment using HFACS. The human factor issues were only noticed when there was an explosion in the university laboratory. The student was the victim of the situation and the deficiency of safety management in the university laboratory.

\section{Acknowledgements}

The authors would like to thank the reviewers of the Journal of Energy and Safety Technology for their insightful review.

\section{References}

[1] Baysari, M.T., McIntosh, A.S., \& Wilson, J.R. (2008). Understanding the human factors contribution to railway accidents and incidents in Australia. Accident Analysis and Prevention, 40, 1750 - 1757.

[2] Madigan, R., Golightly, D., \& Madders, R. (2016). Application of Human Factors and Classification System (HFACS) to UK rail safety of the line incidents. Accident Analysis and Prevention, 97, $122-131$.

[3] Mazaheri, A., Montewka, J., Nisula, J., \& Kujala, P. (2015). Usability of accident and incident reports for evidence-based risk modeling - A case study on ship grounding reports. Safety Science, 76, $202-214$.

[4] Mosaly, P., Mazur, L.M., Miller, S., Eblan, M., \& Jones, E.L. (2013). Applying Human Factors Analysis and Classification System (HFACS) to the analysis of good catches in radiation oncology. Supplement, 87(2), S683. 
[5] Mulcahy, M.B., Young, A., Gibson, J., Hildreth, C., Ashbrook, P., Izzo, R., \& Backus, B. (2013). College and university sector response to the U.S. Chemical Safety Board Texas Tech incident report and UCLA laboratory fatality. Journal of Chemical Health \& Safety, $6-13$.

[6] Reason, J. (1990). Human Error. Cambridge, UK: Cambridge University Press.

[7] Shappell, S.A., \& Wiegmann, D.A. (2000). The Human Factors Analysis and Classification System - HFACS. Springfield, VA: National Technical Information Service.

[8] Shappell, S.A., \& Wiegmann, D.A. (2001). Applying reason: The human factors analysis and classification system (HFACS). Human Factors and Aerospace Safety, 1(1), 59 - 86.

[9] The U.S. Chemical Safety Board [CSB]. (2010). Texas Tech University Laboratory Explosion. Report no. 2010-05-1-TX. Retrieved from http://www.csb.gov/texas-tech-university-chemistry-lab-explosion/. Retrieved date: January 1, 2018.

[10] The U.S. Chemical Safety Board [CSB]. (2011). CSB Releases Investigation into 2010 Texas Tech Laboratory Accident: Case Study Identifies Systemic Deficiencies in University Safety Management Practices. Retrieved from http://www.csb.gov/csb-releases-investigation-into-2010texas-tech-laboratory-accident-case-study-identifies-systemic-deficiencies-in-university-safety-management-practices/. Retrieved date: January $1,2018$. 